\title{
UM PANORAMA DA CATÁLISE NO BRASIL NOS ÚLTIMOS 40 ANOS
}

\author{
Katia Bernardo-Gusmão ${ }^{\mathrm{a}}$, Sibele B. C. Perghere ${ }^{\mathrm{b}, \mathrm{e}}$ E Eduardo N. dos Santos ${ }^{\mathrm{c}, *}$ \\ a'Departamento de Química Inorgânica, Instituto de Química, Universidade Federal do Rio Grande do Sul, 91.510-970 Porto \\ Alegre - RS, Brasil \\ 'Instituto de Química, Universidade Federal do Rio Grande do Norte, 59086-005 Natal - RN, Brasil \\ 'Departamento de Química- ICEx, Universidade Federal de Minas Gerais, 31.270-901 Belo Horizonte - MG, Brasil
}

Recebido em 28/04/2017; aceito em 24/05/2017

\begin{abstract}
UM PANORAMA DA CATÁLISE NO BRASIL NOS ÚLTIMOS 40 ANOS. In this work, the catalysis area in Brazil is analyzed from several perspectives between 1977 and 2017. The conceptual aspects and the area at world level are briefly introduced. A history of the main organizational initiatives of the area is also presented, highlighting the activities of the Catalysis Division of the Brazilian Chemical Society. An overview of the industry situation related to the area, as well as the evolution of scientific production in Brazil, are also discussed.
\end{abstract}

Keywords: Catalysis in Brazil; Catalysis Division of the Brazilian Chemical Society

\section{A RELEVÂNCIA DA CATÁLISE}

Um catalisador é uma substância química que, adicionada em pequenas quantidades, acelera uma reação química sem por ela ser intrinsecamente consumido. A catálise é de vital importância para a indústria química: mais de $80 \%$ dos produtos químicos industriais passam em pelo menos uma etapa de sua fabricação por um processo catalítico. A catálise é particularmente importante para os setores de refino de petróleo, petroquímica, polímeros, agroquímicos, aromas e fragrâncias e fármacos. Uma aplicação já muito importante e em franco crescimento é a de catalisadores para abatimento de poluentes, sobretudo na indústria automotiva. O mercado global de catalisadores, incluindo sua regeneração, girou em torno de 25 bilhões de dólares em 2014, com uma projeção de crescimento anual de cerca de $4 \%$ até $2024 .{ }^{1}$

A utilização de processos catalíticos é explicitamente um dos doze princípios da Química Verde, mas correlaciona-se intimamente com vários outros tais como: maior seletividade atômica, economia de energia e rotas sintéticas mais curtas. ${ }^{2}$ Devido às pressões sociais e governamentais por processos mais limpos, a introdução de novos processos industriais baseados em catálise podem ser esperados para os próximos anos.

Na catálise industrial, há uma divisão clássica em catálise heterogênea e catálise homogênea. Na catálise heterogênea os reagentes e catalisadores encontram-se em fases distintas e a reação ocorre na interface. Catalisadores típicos são metais com alta área específica, metais suportados em sólidos de alta área específica, óxidos metálicos, zeólitas, argilas, haletos metálicos, entre outros. Esta classe corresponde a cerca de $74 \%$ em volume do mercado de catalisadores e está mais associada à indústria de refino do petróleo, da petroquímica, da química de base e de abatimento de poluentes. Na catálise homogênea, o catalisador e reagentes encontram-se em uma mesma fase. Catalisadores típicos desta classe são compostos ácidos ou básicos de baixo peso molecular e complexos de metais de transição utilizados em solução. Apesar da menor fração em volume do mercado, esta classe é utilizada intensamente na produção de compostos de maior valor agregado em vários setores da química fina, com destaque ao de fragrâncias e aromas, agroquímico e farmacêutico.

*e-mail: nicolau@ufmg.br

"e-mail: sibelepergher@gmail.com
Devido à sua grande importância tecnológica e científica é notável, porém não surpreendente, que os primeiros anos deste milênio tenham testemunhado nada menos que quatro prêmios Nobel em química na temática de catálise, sendo que três deles envolvem a catálise por complexos de metais de transição. ${ }^{3} \mathrm{O}$ prêmio de 2001 foi concedido a Willian S. Kowles (Monsanto Company/ EUA), Ryoji Noyori (Nagoya University/ Japan) e K. Barry Sharpless (The Scripps Research Institute /USA) por seus avanços e desenvolvimentos na síntese orgânica enantiosseletiva utilizando complexos de metais de transição com ligantes quirais como catalisadores, o que permitiu a síntese industrial de produtos homoquirais de alto valor agregado, tais como componentes de fragrâncias e fármacos. O Nobel em Química de 2005 foi atribuído a Yves Chauvin (IFP/ França), Robert H. Grubbs (CALTECH/EUA) e Richard R. Schrock (MIT/EUA), por suas contribuições para a metátese de olefinas, uma reação que permitiu significativos avanços na síntese orgânica industrial. O primeiro contribuiu decisivamente para a elucidação mecanística desta reação e os últimos desenvolveram catalisadores moleculares de estrutura bem definida, que permitiram a ampla utilização desta reação na pesquisa em síntese orgânica, assim como a sua utilização industrial no setor farmacêutico, de bio-refinarias e de polímeros especiais. O prêmio Nobel em Química de 2007 foi concedido a Gerhard Ertl (Fritz-Haber-Institut der Max-Planck-Gesellschaft/ Alemanha) por seus estudos inovadores na química de superfícies, um assunto fulcral para a catálise. Através desses estudos, foi possível melhorar a compreensão da síntese da amônia pelo processo HaberBosch, considerado por muitos a invenção mais relevante do século 20. O prêmio de 2010 foi concedido a Richard F. Heck (University of Delaware/EUA), Ei-ichi Negishi (Purdue University/EUA) e Akira Suzuki (Hokkaido University/Japão) pelo desenvolvimento dos acoplamentos cruzados catalisados por complexos de paládio. Essa metodologia permite a formação de uma ligação carbono-carbono entre fragmentos orgânicos complexos (synthons) para a construção de moléculas de importância biológica, de maneira versátil. As aplicações incluem síntese de agroquímicos, ingredientes farmacologicamente ativos e insumos para a indústria eletrônica.

A importância da área é também refletida no número e na qualidade de periódicos científicos dedicados ao tema, como pode ser visto na Tabela 1. Os índices de impacto dos periódicos ACS Catalysis, Applied Catalysis B: Environmental e Journal of Catalysis, estão entre os mais altos da área de química. 
Tabela 1. Periódicos de maior impacto especializados em catálise

\begin{tabular}{|c|c|c|c|}
\hline Revista & Editora & Ano de Início & Fator de Impacto $(2015 / 2016)$ \\
\hline ACS Catalysis & $\mathrm{ACS}$ & 2011 & 9.307 \\
\hline Applied Catalysis B, Environmental & Elsevier & 1992 & 8.328 \\
\hline Journal of Catalysis & Elsevier & 1962 & 7.354 \\
\hline Advanced Synthesis \& Catalysis & Wiley & 1999 & 6.453 \\
\hline Catalysis Science \& Technology & RSC & 2011 & 5.287 \\
\hline ChemCatChem & Wiley & 2009 & 4.724 \\
\hline Advances in Catalysis & Elsevier & 1948 & 4.5 \\
\hline Catalysis Today & Elsevier & 1987 & 4.312 \\
\hline Applied Catalysis. A, General & Elsevier & 1991 & 4.012 \\
\hline Journal of Molecular Catalysis A: Chemical & Elsevier & 1995 & 3.958 \\
\hline Catalysis Communications & Elsevier & 2000 & 3.389 \\
\hline Topics in Catalysis & Springer & 1994 & 2.355 \\
\hline Catalysis Letters & Springer & 1988 & 2.294 \\
\hline Journal of Molecular Catalysis B: Enzymatic & Elsevier & 1995 & 2.189 \\
\hline
\end{tabular}

Fonte: www-periodicos-capes-gov-br e www.scijournal.org.

\section{VISÃO HISTÓRICA DA CATÁLISE NO BRASIL}

A instalação dos parques industriais de refino de petróleo e petroquímica no Brasil se deu nos anos 1960 e 1970 e para essas indústrias a catálise é um tema essencial. Datam desta época os primeiros esforços sistemáticos para o desenvolvimento da catálise como área de pesquisa no Brasil. ${ }^{4} \mathrm{O}$ primeiro Seminário Brasileiro de Catálise foi organizado em 1981 e desde então vem acontecendo a cada dois anos. Inicialmente o encontro foi organizado pela Comissão de Catálise do Instituto Brasileiro do Petróleo (IBP). Em 1997 o encontro passou a chamar-se Congresso Brasileiro de Catálise e em várias edições foi co-organizado com o Congresso de Catálise do Mercosul. No ano de 2017 será realizada a sua $19^{\mathrm{a}}$ edição em conjunto com o $9^{\circ}$ Congresso de Catálise do Mercosul.

Paralelamente a este esforço organizacional, em 1983 foi criado um programa de apoio específico à área junto ao $\mathrm{CNPq}$, o Programa Nacional de Catálise, que perdurou 4 anos e foi bastante importante para o desenvolvimento da área junto às universidades. Programas de cooperação com a outros países, com destaque para a França e para a Espanha, que capitaneou o Programa CYTED, foram também importantes para a formação de recursos humanos na área.

Apesar dos esforços e das atividades desenvolvidas na área pela Comissão de Catálise do IBP e das várias edições do Seminário Brasileiro de Catálise, não havia até 1995 uma sociedade científica brasileira dedicada ao tema. A pesquisa nesta área tem ampla aderência a temas da Química como a cinética química, a química de sólidos e superfícies, a química supramolecular, a química de coordenação e organometálica, a química de materiais, a química teórica, entre outros. Quando transposta ao cenário industrial, naturalmente os objetos de estudo da Engenharia Química tais como os fenômenos de transporte de massa e energia, materiais, entre outros, adquirem importância fundamental. Assim, a catálise tem uma forte interface entre a Química e a Engenharia Química.

Quando a Sociedade Brasileira de Química começou a organizar-se em divisões científicas, foi natural a criação da Divisão de Catálise da SBQ, dada a importância da área e a sua adesão à Química, o que se deu em 1995. Entretanto, naquela ocasião, a maioria da pesquisa na área de catálise, sobretudo em catálise heterogênea, se dava na comunidade de engenharia química, cujos membros não frequentavam as reuniões da SBQ. Os frequentadores do Seminário Brasileiro de
Catálise julgaram oportuna a criação de uma sociedade científica específica para a catálise e assim foi fundada em 1997 a Sociedade Brasileira de Catálise (SBCat). ${ }^{4} \mathrm{~A}$ integração dessas duas entidades se deu pela concessão de um assento no conselho consultivo da SBCat ao diretor da Divisão de Catálise da SBQ. As atividades desenvolvidas pela Sociedade Brasileira de Catálise podem ser encontradas na página da internet desta sociedade (www.sbcat.org).

\section{Atividades da Divisão de Catálise da SBQ}

Desde sua fundação em 1995, a Divisão de Catálise da SBQ vem tendo participação ativa nas Reuniões Anuais da Sociedade Brasileira de Química (RASBQ). ${ }^{5}$ Esteve entre as pioneiras na organização de oficinas (workshops) pré-reunião, cujos temas estão listados na Tabela 2.

Preocupada com a formação de recursos humanos e de divulgação da área, a Divisão tem a tradição de oferecer minicursos durante as reuniões anuais, que cobrem temas básicos e avançados relacionados à área, como pode ser visto na Tabela 3.

Nas RASBQ podemos observar muitas conferências plenárias com pesquisadores da área de Catálise, o que demonstra a relevância do tema para a SBQ. Muitos destes convites são frutos da forte inserção nacional e internacional que os membros da comunidade catalítica apresentam. Alguns exemplos destas Conferências nestes últimos anos estão listados na Tabela 4.

Devido ao seu caráter multidisciplinar, a Divisão de Catálise tem co-organizado sessões coordenadas com as divisões de Síntese Orgânica, de Materiais, entre outras.

\section{A catálise na indústria química brasileira}

Apesar de comparativamente pequeno, o segmento de catalisadores tem enorme importância estratégica para os vários segmentos da indústria química e cada vez mais este mercado está se concentrando em torno de um número pequeno de atores internacionais. ${ }^{6}$

Até o início da década de 1990, várias indústrias petroquímicas mantinham centros de $\mathrm{P} \& \mathrm{D}$ onde processos catalíticos e o estudo de catalisadores tinham um papel de destaque, tendo as universidades um papel ativo neste desenvolvimento. Após a abertura do mercado, vários desses centros foram desativados. Uma notável exceção foi a 
Tabela 2. Oficinas organizadas pela Divisão de Catálise da SBQ

\begin{tabular}{|c|c|}
\hline RASBQ & Tema \\
\hline $39^{\mathrm{a}}$ & $\begin{array}{l}\text { Transformações inteligentes de resíduos para obtenção de catali- } \\
\text { sadores e materiais estratégicos }\end{array}$ \\
\hline $38^{\mathrm{a}}$ & Materiais e Processos Catalíticos Verdes \\
\hline $37^{\mathrm{a}}$ & $\begin{array}{l}\text { Processos de transformação química como ferramentas na solução } \\
\text { de problemas ambientais }\end{array}$ \\
\hline $36^{\mathrm{a}}$ & Processos eletrocatalíticos \\
\hline $35^{\mathrm{a}}$ & Catálise para a Transformação de Biomassa \\
\hline $34^{\mathrm{a}}$ & Catálise na era do Pré-Sal \\
\hline $33^{\mathrm{a}}$ & Síntese Caracterização e Aplicações de Nanocatalisadores \\
\hline $32^{\mathrm{a}}$ & Catálise a Serviço da Química Verde \\
\hline $31^{\mathrm{a}}$ & Algumas contribuições da Catálise para uma Biorefinaria \\
\hline $30^{\mathrm{a}}$ & Catálise no Setor de Energia \\
\hline $29^{\mathrm{a}}$ & Catálise na Petroquímica \\
\hline $28^{\mathrm{a}}$ & Catálise na Oleoquímica \\
\hline $25^{\mathrm{a}}$ & Química sol-gel: Catálise e Novos Materiais \\
\hline $23^{\mathrm{a}}$ & $2^{\circ}$ Workshop em Catálise Homogênea* \\
\hline $21^{\mathrm{a}}$ & $1^{\circ}$ Workshop em Catálise da SBQ \\
\hline
\end{tabular}

* Um evento satélite à $23^{\text {a }}$ RASBQ organizado em Ouro Preto-MG em maio de 2000.

indústria de poliolefinas, que dependia de grades especiais de polímeros de maior valor agregado para se manter competitiva. A atividade de $P \& D$ com parcerias com as universidades neste segmento foi mais longeva. Mais recentemente, várias parcerias foram estabelecidas na área de biocombustíveis.

O tamanho do mercado de catalisadores no Brasil não chega a ser convidativo para a produção industrial. Muitos dos desenvolvimentos foram motivados por dificuldades de importações e por questões estratégicas. Foram pioneiros na produção industrial de catalisadores no Brasil a OXITENO e a Fábrica Carioca de Catalisadores. A OXITENO deu início à produção na década de 1980 especializando-se em catalisadores para a geração de hidrogênio e para a oxidação do etileno a óxido de etileno. Merece destaque o desenvolvimento de catalisadores para processos alcoolquímicos motivados pelo Programa Nacional de Álcool. Por volta de 2012, seguindo uma tendência mundial, o negócio de catalisadores da OXITENO foi adquirido pela CLARIANT, que, entretanto, mantém o centro de pesquisa no Brasil.

A decisão da construção da Fábrica Carioca de Catalisadores foi de caráter estratégico, motivado pela interrupção de fornecimento de catalisadores de craqueamento de petróleo à Argentina durante o conflito das Malvinas/Falklands em 1984. A empresa, formada uma parceria entre a PETROBRAS e a Albemale Corporation, dedica-se à produção de catalisadores para refino de petróleo.

A produção de catalisadores automotivos no Brasil foi iniciada pela Degussa, agora UMICORE. A BASF também produz catalisadores automotivos em Indaiatuba-SP. Além disso, a UMICORE tem uma unidade de produção de sais de metais nobres que são usados como catalisadores ou são seus insumos.

Certos processos industriais baseados em catalisadores merecem menção. A produção do fungicida Boscalid é feita pela BASF em Guaratinguetá-SP utilizando catalisadores de paládio em solução (catálise homogênea). Este é o maior processo industrial em nível mundial que emprega o acoplamento de Suzuki.

Grande parte do negócio da BRASKEM depende de catalisadores para polimerização que são produzidos para consumo interno. A desidratação do etanol a etileno e a sua conversão a polietileno (polietileno verde) são praticadas pela mesma empresa em Triunfo-RS na escala de 200 mil toneladas/ano. Em uma iniciativa análoga, o projeto de um complexo de grande porte no Triângulo Mineiro envolvendo as companhias Mitsui e Dow para a produção dedicada de etanol integrada a uma planta para a desidratação/ polimerização foi postergada. Uma possível razão foi o desenvolvimento da exploração de gás de xisto nos Estados Unidos, rico em hidrocarbonetos leves, consistindo em uma alternativa barata para olefinas. Entretanto, a planta para a produção de etanol foi construída pela Mitsui.

\section{AVALIAÇÃo DA PRODUÇÃo CIENTÍFICA EM CATÁLISE NO BRASIL}

Para avaliar a evolução da produção científica brasileira na área, foi utilizada a base de dados Web of Science (Thomson Reuters), com os seguintes critérios de busca: Título: Catalyst ${ }^{*}$ or catalysed or catalyzed - Tópico: catalyst*; Período: 1977-2017; Tipo de documento: article; Pais: Brasil. Embora lógicos, esses critérios são arbitrários e

Tabela 3. Minicursos organizados pela Divisão de Catálise (2007-2016).

\begin{tabular}{|c|c|c|}
\hline RASBQ & Título & Ministrantes \\
\hline $39^{\mathrm{a}}$ & Propriedades e aplicações dos nanocatalisadores & $\begin{array}{c}\text { Maria do Carmo R.S. Varela (UFBA) } \\
\text { Emilia Celma de O. Lima (UFG) }\end{array}$ \\
\hline $38^{\mathrm{a}}$ & Catálise em Processos Industriais & $\begin{array}{c}\text { Mario Meneghetti (UFAL) } \\
\text { Simoni M. P. Meneghetti (UFAL) }\end{array}$ \\
\hline $37^{\mathrm{a}}$ & A adsorção em superfícies e as aplicações em catálise & Michele Oberson de Souza (UFRGS) \\
\hline $36^{\mathrm{a}}$ & Catálise e química verde & Dalmo Mandelli (UFABC) \\
\hline $35^{\mathrm{a}}$ & As reações catalíticas que fizeram história & Eduardo Nicolau dos Santos (UFMG) \\
\hline $34^{\mathrm{a}}$ & Conceitos e aspectos gerais em Catálise & Wagner Alves Carvalho (UFABC) \\
\hline $33^{\mathrm{a}}$ & Técnicas analíticas aplicadas à Catálise & $\begin{array}{l}\text { Liane Márcia Rossi (IQUSP) } \\
\text { Giovanna Machado (UCS) }\end{array}$ \\
\hline $32^{\mathrm{a}}$ & Catálise Industrial & $\begin{array}{l}\text { Katia Bernardo Gusmão (UFRGS) } \\
\text { Sibele B. C. Pergher (URI) }\end{array}$ \\
\hline $31^{\mathrm{a}}$ & Fundamentos da Catálise & $\begin{array}{l}\text { Mario Meneghetti (UFAL) } \\
\text { Sibele B. C. Pergher (URI) }\end{array}$ \\
\hline $30^{\mathrm{a}}$ & Fundamentos de Catálise & $\begin{array}{l}\text { Mario Meneghetti (UFAL) } \\
\text { Sibele B. C. Pergher (URI) }\end{array}$ \\
\hline
\end{tabular}


Tabela 4. Conferências Plenárias em Catálise nas RASBQ (período: 2006-2016).

\begin{tabular}{|c|c|c|}
\hline RASBQ & Título & Conferencista \\
\hline $39^{\mathrm{a}}$ & Principais desafios catalíticos na implementação de uma Biorrefinaria & Eduardo Falabella Sousa-Aguiar (UFRJ) \\
\hline $39^{\mathrm{a}}$ & $\begin{array}{l}\text { Catalysis for the Synthesis of Complex Organic Molecules and Industrial Chemicals: } \\
\text { What are the Differences }\end{array}$ & Matthias Beller (Leibnitz Institute for Catalysis, Alemanha) \\
\hline $39^{\mathrm{a}}$ & Magadiite: possibilities of a lamellar solid with unknown crystalline structure & Heloise de Oliveira Pastore (UNICAMP) \\
\hline $39^{\mathrm{a}}$ & Continuous photoredox catalysis in continuous-flow reactors & Timothy Noël (Eindhoven University of Technology, Holanda) \\
\hline $38^{\mathrm{a}}$ & Modeling Approaches Reality: Structure and Reactivity of Supported Catalysts & Francesc Illas (Universitat de Barcelona, Espanha) \\
\hline $38^{\mathrm{a}}$ & Liquid-phase catalytic conversion of lignocellulosic biomass to fuels and chemicals & James A. Dumesic (University of Wisconsin, Estados Unidos) \\
\hline $37^{\mathrm{a}}$ & Metal catalysis: New reactions and new opportunities for organic synthesis & Timothy J. Donohoe (University of Oxford, Inglaterra) \\
\hline $35^{\mathrm{a}}$ & Materials design for catalysis & Avelino Corma (ITQ/UPV - Espanha) \\
\hline $34^{\mathrm{a}}$ & $\begin{array}{l}\text { Geração de } \mathrm{H}_{2} \text { através de reações de reforma: desafios para o entendimento dos } \\
\text { catalisadores in situ }\end{array}$ & José Maria Correa Bueno (UFSCar) \\
\hline $33^{\mathrm{a}}$ & $\begin{array}{l}\text { Desenvolvimento de sistemas catalíticos para a produção de olefinas leves e nano- } \\
\text { compósitos poliméricos }\end{array}$ & Osvaldo de Lázaro Casagrande Jr. (UFRGS) \\
\hline $32^{\mathrm{a}}$ & Biocatalysis, Green Chemistry \& Sustainability & Roger A. Sheldon (Delft University, Holanda) \\
\hline $31^{\mathrm{a}}$ & Contribuições da Catálise no aproveitamento da biomassa & Ulf F. Schuchardt (UNICAMP) \\
\hline $31^{\mathrm{a}}$ & Catalytic activation of unsaturated and saturated hydrocarbons & $\begin{array}{l}\text { Helmut Guido Alt (Universidade de Bayreuth, Germany, } \\
\text { DAAD) }\end{array}$ \\
\hline $29^{\mathrm{a}}$ & Electrocatalysts for fuel cell applications & Hector Abruña (Cornell Univ, EUA) \\
\hline
\end{tabular}

os números a seguir devem ser vistos como tendências, ao invés de uma classificação sistemática e rigorosa. Em uma busca realizada em 23/04/2017 com esses critérios foram localizados 452.179 artigos em nível mundial com 6.565 artigos $(1,45 \%)$ listando o Brasil como pais da produção. O Brasil figura em $16^{\circ}$ lugar em nível mundial e primeiro na América Latina. Para comparação, o México conta com $3.449 \operatorname{artigos}\left(24^{\circ}\right)$ e a Argentina $2.922 \operatorname{artigos~}\left(28^{\circ}\right)$. A evolução no número de artigos publicados listando o Brasil como pais da produção e o correspondente número de citações podem ser acompanhados na Figura 1. O gráfico reflete um aumento linear na produção a partir dos anos 1990 a uma taxa de crescimento de aproximadamente 20 artigos por ano. O número de citações aumentou exponencialmente no período. Como esta é uma tendência mundial devido às facilidades introduzidas pelas publicações eletrônicas e bases de dados, foram analisados separadamente o período inicial e o final da pesquisa. Quando a pesquisa é limitada ao período inicial 1977-1987 encontramos 21.901 artigos no mundo, sendo 45 no Brasil (0,20\%), o que deixa o país em $39^{\circ}$ lugar, atrás da Argentina $\left(30^{\circ}\right)$ e da Venezuela $\left(33^{\circ}\right)$. Quando a pesquisa é limitada à última década (2007 a 2017), encontramos 266.005 artigos em nível mundial, sendo 4.814 (1,80\%) brasileiros, posicionado o país em $15^{\circ}$ lugar, à frente do México $\left(26^{\circ}\right)$ e da Argentina $\left(32^{\circ}\right)$. Portanto, a participação científica do Brasil em nível mundial na área de catálise evoluiu sobremaneira, mas a mudança de posicionamento da Venezuela na última década $\left(59^{\circ}\right)$ é um alerta para os efeitos nefastos da interrupção de financiamento da pesquisa científica.

As contribuições sobre o tema em Química Nova e no Journal of the Brazilian Chemical Society (JBCS) no período compreendido entre 2004 e 2006 foram avaliadas e comparadas com jornais especializados em catálise. ${ }^{7}$ Recentemente, um número do JBCS foi dedicado ao tema de catálise. ${ }^{4}$ Os autores dedicaram os trabalhos ao Professor Roberto Fernando de Souza do Instituto de Química da UFRGS (homenagem póstuma), que foi um grande entusiasta e promotor do tema no Brasil.

$\mathrm{O}$ aumento da visibilidade internacional do país na área propiciou a atração de eventos científicos internacionais, como pode ser observado na Tabela 5.
A catálise por complexos de metais de transição (catálise homogênea) está proporcionalmente mais representada junto à Divisão de Catálise da SBQ. Entretanto, esta área tem sido amplamente assimilada pela comunidade de síntese orgânica. Os Brazilian Meetings in Organic Synthesis (BMOS) têm atraído importantes nomes da comunidade científica internacional da área, incluindo prêmios Nobel como Suzuki, e Grubbs. Também foi realizado em Angra dos Reis em 2015 o $5^{\circ}$ Simpósio de Química de Coordinación y Organometalica, no qual a catálise por complexos de metais de transição foi um tema central e atraiu vários nomes altamente conceituados na área.

\section{CONCLUSÕES}

A mudança de perfil econômico com a globalização levou a inúmeras fusões e incorporações na indústria química. Vários países líderes neste setor têm visto suas empresas tradicionais sendo incorporadas por empresas transnacionais e seus centros de pesquisa sendo deslocados a outras partes do mundo. Fica a pergunta que transcende o campo específico deste artigo: vale a pena privilegiar o apoio à pesquisa básica em áreas de interesse industrial em países com o perfil do Brasil? A resposta é não é tão óbvia, mas se este investimento não for feito, o pais certamente não terá condições nem agora e nem no futuro de ambicionar uma posição minimamente relevante no cenário internacional. Uma diminuição no nível de investimentos seria desastrosa para as gerações futuras, pois a formação de recursos humanos e o conhecimento acumulado seriam perdidos. Para que a pesquisa reflita na apropriação de valor agregado na cadeia produtiva de insumos químicos pelo país, os agentes públicos devem não só aumentar o nível de fomento à pesquisa básica, pelo menos em um nível compatível com os países detentores de tecnologia, mas também deve estimular programas de incentivo consistentes e de longo prazo que contemplem também a transição do conhecimento da academia ao setor produtivo, sobretudo em nichos de mercado em que o Brasil tenha um bom posicionamento estratégico. No contexto da indústria química, o Brasil ocupa uma posição privilegiada na produção de insumos químicos bio-renováveis por seu notável potencial agrícola. Este mercado foi de 49 bilhões de dólares em 2014 e deverá crescer 

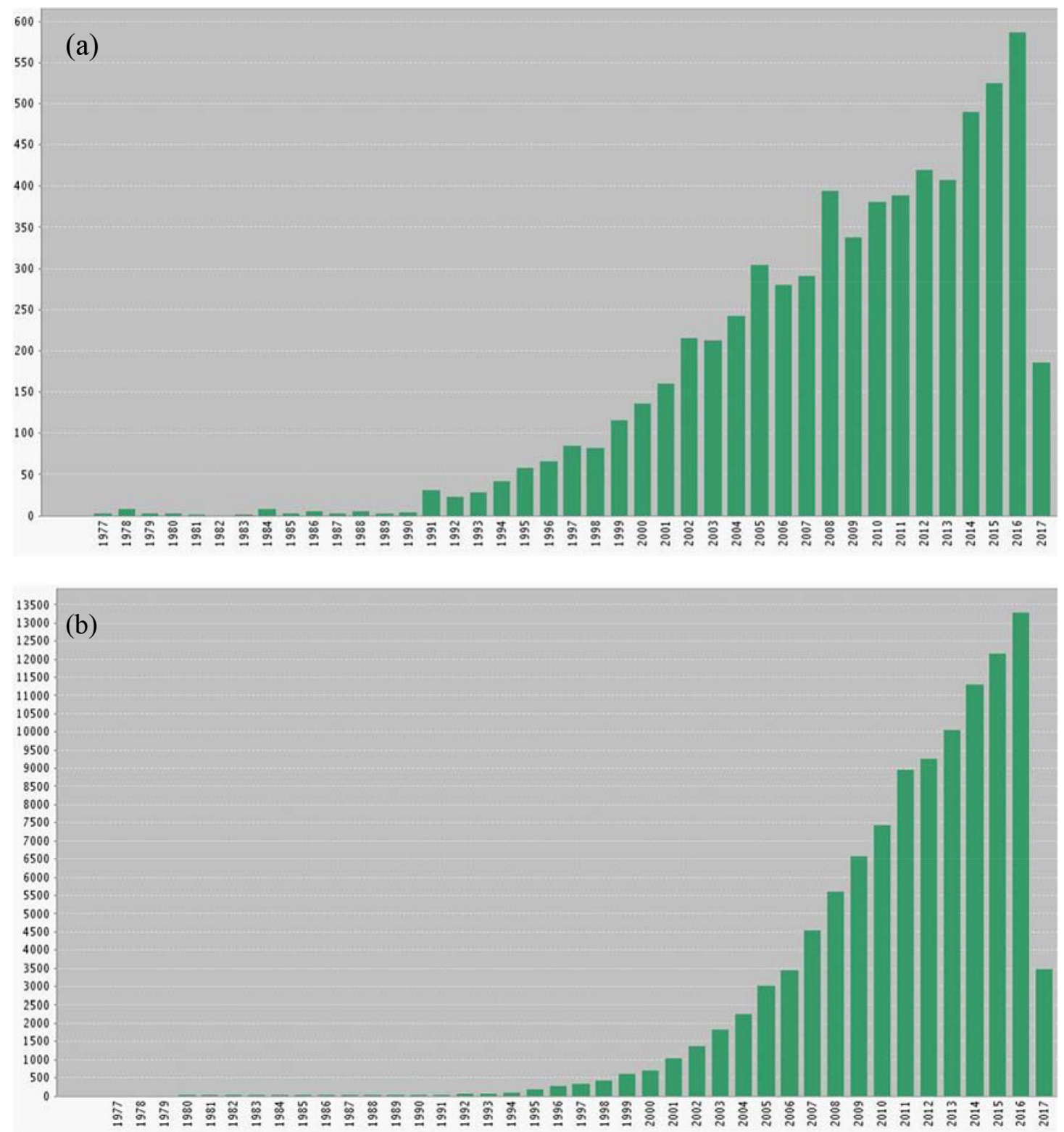

Figura 1. Evolução da produção científica em catálise no Brasila (a) número de artigos, (b) Número de citações. ${ }^{a}$ critérios de busca no Web of Science: Título: Catalyst* or catalysed or catalyzed + Tópico: catalyst*; Período: 1977-2017; Tipo de documento: article; Pais: Brasil

Tabela 5. Eventos Internacionais relacionados à Catálise realizados no Brasil (período: 2006-2017)

\begin{tabular}{lcc}
\hline Ano & Evento & Local \\
\hline 2017 & $8^{\text {th }}$ International Symposium on Acid-Base Catalysis, ABC-8 & Rio de Janeiro/RJ \\
2017 & IX Congresso de Catálise do Mercosul & Ouro Preto/MG \\
2016 & $18^{\text {th }}$ International Zeolite Conference, 18th IZC & Rio de Janeiro/RJ \\
2015 & $3^{\text {o }}$ Congresso Internacional de Catálise para Biorrefinarias, $3^{\text {o }}$ CatBior & Rio de Janeiro/RJ \\
2013 & VII Congresso de Catálise do Mercosul & Gramado/RS \\
2009 & V Congresso de Catálise do Mercosul & Armação de Búzios/RJ \\
2007 & 8th Natural Gas Conversion Symposium & Natal/RN \\
2006 & XX Simpósio Ibero-americano de Catálise & Gramado/RS \\
\hline
\end{tabular}

em uma taxa de $11,5 \%$ ao ano nos próximos cinco anos. ${ }^{8} \mathrm{O}$ bioetanol é o produto mais comercializado entre os renováveis e a revitalização da cadeia produtiva de insumos químicos a partir do etanol parece uma alternativa a ser estudada. A produção de insumos a partir de materiais ligno-celulósicos e o avanço na cadeia produtiva baseada em óleos essenciais, fornecendo não apenas a matéria-prima, mas também produtos purificados e transformados de maior valor agregado, parecem ser promissores. A produção de insumos químicos de alto valor agregado a partir de óleos vegetais (bio-refinarias baseadas na tecnologia de metátese) pode ser muito mais lucrativa e sustentável 
que a produção de biodiesel. Em todos os setores mencionados, a catálise ocupa uma posição central e programas especiais de incentivo à área seriam muito oportunos para alavancar este setor industrial.

\section{REFERÊNCIAS}

1. https://www.bccresearch.com/market-research/chemicals/catalystregeneration-global-markets-chm046c.html, acessada em abril 2017.

2. Sheldon, R. A.; J Mol Catal A: Chemical 2016, 422, 3.
3. https://www.nobelprize.org/nobel_prizes/chemistry/laureates/, acessada em abril de 2017.

4. Schmal, M.; J Brazil Chem Soc 2014, 25, 2135.

5. Dupont, J.; Quim Nova 2002, 25, 12.

6. http://www.abifina.org.br/arquivos/revistas/facto31.pdf, acessada em abril 2017.

7. dos Santos, E. N.; Lago, R. M.; Quim Nova 2007, 30, 1480.

8. http://www.marketsandmarkets.com/PressReleases/renewable-chemical. asp, acessada em abril 2017. 